\title{
Are schoolchildren less infected if they have good knowledge about parasitic worms? A case study from rural Côte d'Ivoire
}

\author{
Marta S. Palmeirim ${ }^{1,2,3}$, Mamadou Ouattara ${ }^{4}$, Clémence Essé5, ${ }^{5,6}$, Véronique A. Koffi ${ }^{5,6}$, Rufin K. Assaréé,6, \\ Eveline Hürlimann ${ }^{1,2}$, Jean T. Coulibaly ${ }^{1,2,4,6}$, Nana R. Diakité ${ }^{4}$, Kouassi Dongo ${ }^{4,6}$, Bassirou Bonfoh ${ }^{1,2,6}$, \\ Jürg Utzinger ${ }^{1,2}$, Eliézer K. N'Goran ${ }^{4,6}$ and Giovanna Raso ${ }^{1,2^{*}}$ (iD
}

\begin{abstract}
Background: Parasitic worms (helminths) are common infections in low- and middle-income countries. For most helminth species, school-aged children are at highest risk of infection and morbidity, such as impaired cognitive and physical development. Preventive chemotherapy is the current mainstay for helminthiases control. Sanitation improvement and hygiene-related education are important complementary strategies, which act by altering children's behaviour. However, little is known about the effect of improved knowledge on the risk of helminth infection. The aim of this study was to assess the potential influence of knowledge that children acquired at home or in school, without any specific health education intervention, on helminth infections.
\end{abstract}

Methods: In May 2014, we conducted a cross-sectional survey in western Côte d'Ivoire. A total of 2498 children, aged 9-12 years, were subjected to three consecutive stool examinations using duplicate Kato-Katz thick smears to determine infections with soil-transmitted helminths and Schistosoma mansoni. Additionally, children were interviewed to assess their knowledge about helminth infections. Four knowledge scores were constructed by factor analysis; one, reflecting general knowledge about helminths and three manifesting helminth species-specific knowledge. The effect of general and specific knowledge on children's helminth infection status was determined using meta-analysis.

Results: Children who scored high in the hookworm-specific knowledge were less likely to be infected with hookworm but no association was found for the other helminth species. Moreover, greater general knowledge was not associated with lower odds of being infected with any helminth species. Most of the children interviewed believed that the effect of preventive chemotherapy is permanent, and hence, re-treatment is not necessary.

Conclusions: Specific knowledge about different types of helminths might not suffice to induce behavioural change which in turn reduces infection and reinfection with helminths. Health education interventions should strive to strengthen the perception of risk and to clarify the true benefit of preventive chemotherapy.

Keywords: Soil-transmitted helminths, Schistosoma mansoni, Health education, Knowledge, Risk perception, Awareness, Côte d'Ivoire

\footnotetext{
* Correspondence: giovanna.raso@swisstph.ch

${ }^{1}$ Swiss Tropical and Public Health Institute, Basel, Switzerland

${ }^{2}$ University of Basel, Basel, Switzerland

Full list of author information is available at the end of the article
}

(c) The Author(s). 2018 Open Access This article is distributed under the terms of the Creative Commons Attribution 4.0 International License (http://creativecommons.org/licenses/by/4.0/), which permits unrestricted use, distribution, and reproduction in any medium, provided you give appropriate credit to the original author(s) and the source, provide a link to the Creative Commons license, and indicate if changes were made. The Creative Commons Public Domain Dedication waiver (http://creativecommons.org/publicdomain/zero/1.0/) applies to the data made available in this article, unless otherwise stated. 


\section{Background}

Parasitic worms, particularly soil-transmitted helminths and schistosomes, are widespread. Indeed, an estimated 1.5 billion people are infected, mainly in low- and middle-income countries [1, 2]. Helminthiases cause considerable burden, including physical and intellectual growth retardation among preschool- and school-aged children [3, 4]. Despite their negative impact on public health, educational attainment, social and economic development, helminthiases are often neglected [5, 6]. Helminthiases account for the largest burden among the neglected tropical diseases [7] and are governed by inadequate water supply and sanitation, improper hygiene habits, crowded living conditions, difficult access to health care and low levels of education $[2,8]$.

Key strategies recommended by the World Health Organization (WHO) for the prevention, control and elimination of helminthiases include preventive chemotherapy (periodic deworming of school-aged children and other high risk groups), and water and sanitation supply supported by personal hygiene and health education [9]. Although effective in the short-term, preventive chemotherapy does not prevent reinfection and, providing adequate sanitation and clean water alone, do not necessarily mean these measures will be adequately used, in which case prevalence and intensity of infection may not decrease in the long run [10-12]. Improved knowledge through health and hygiene-related education might induce behavioural change, which in turn might result in reduced exposure to helminth infections, thus avoiding or delaying reinfection [13-15]. Indeed, the gains of preventive chemotherapy, water supply and sanitation improvement can be reinforced through enhanced knowledge [16, 17].

Although this topic has gained traction in recent years, relatively little research has investigated the effect of health education interventions on the prevalence, intensity and re-infection patterns of soil-transmitted helminths [18-21]. However, results reported in the literature are inconsistent. While some studies have found that health education was associated with a decrease in helminth infection $[17,18$, $21]$, other studies failed to detect such an association [19, 20]. This discrepancy might be explained by specific geographic, social, cultural and infrastructure conditions under which the effectiveness of health education was evaluated. Hence, there is a need for empirical studies assessing the effect of knowledge on helminth infection rates. Research pursued in different settings will deepen our understanding of how improved knowledge results in reduced rates of helminth infection.

A limitation of the available information on the effect of knowledge on helminth infection is that most studies carried out thus far focused on the follow-up of specific health education interventions, which are usually meant to not only increase knowledge but also to raise awareness and demystify local myths $[17,18,20]$. However, typically, health-related knowledge that children receive does not come from such specific interventions, but is rather "background" knowledge obtained at home or in school. The level of this "background" knowledge is likely to vary from one child to another and across settings.

In the current study, we assessed the importance of "background" knowledge that children acquired at home or in school, without any specific health education intervention on helminth infections. Our hypothesis was that children's "background" knowledge regarding soil-transmitted helminths and Schistosoma mansoni infections influences their odds of infection, and hence, children with adequate knowledge have better health and hygiene-related behaviours, which in turn results in lower helminth infection levels.

\section{Methods}

\section{Study site and study design}

The study was carried out in four regions of western Côte d'Ivoire: Cavally, Guémon, Haut-Sassandra and Tonkpi (Fig. 1). In this part of Côte d'Ivoire, people are mainly engaged in subsistence agriculture [22]. Previous studies in this area have shown that $S$. mansoni and hookworm infections are highly endemic $[23,24]$. The study was implemented in 25 schools that are part of a large 5-year operational research project pertaining to sustaining the control of schistosomiasis, facilitated by the Schistosomiasis Consortium for Operational Research and Evaluation (SCORE) [25, 26]. Children in these schools have been receiving an annual dose of praziquantel (40 mg/kg) and albendazole (400 mg) since 2012. An observation of sanitary conditions performed one year after the implementation of the current study showed that, among the 25 schools investigated, soap for washing hands after defecation was only available in three schools (12\%), access to clean water from a pump was available in 10 schools (40\%) and latrines were available in 17 schools (68\%).

In May 2014, about 100 children, aged 9-12 years, were selected in each of the 25 schools. The study consisted of two surveys: (i) a cross-sectional parasitological survey to assess children's infection with helminths; and (ii) a questionnaire survey to determine children's knowledge about helminthiases. After the parasitological survey, all school-aged children (5-15 years) of the study localities, including those not participating in our study, were treated with albendazole $(400 \mathrm{mg})$ against soil-transmitted helminthiasis and praziquantel $(40 \mathrm{mg} / \mathrm{kg})$ against schistosomiasis by the national control programme.

\section{Parasitological survey}

Each participant was provided with three empty plastic containers that contained unique identification numbers and was invited to submit three stool samples over 


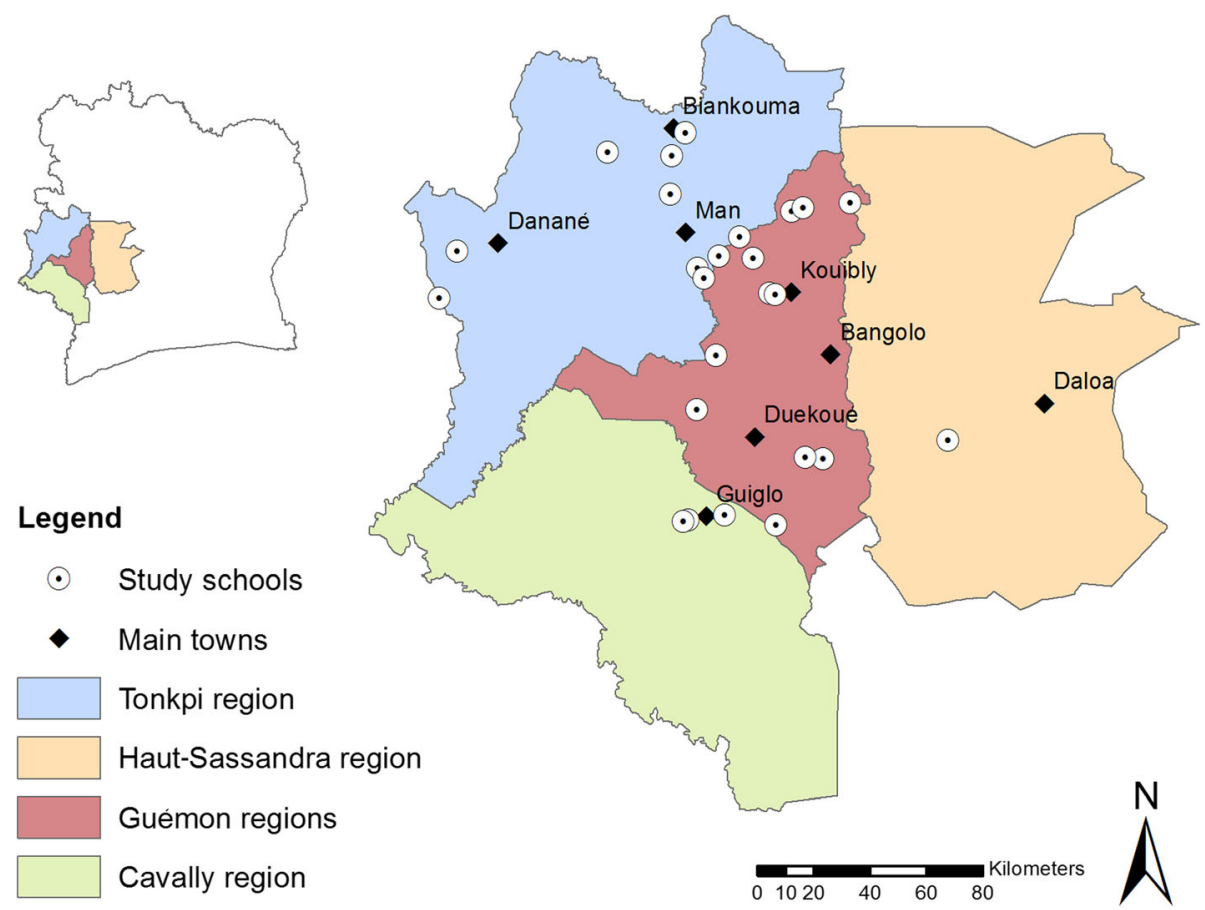

Fig. 1 Map of Côte d'Ivoire with the four regions and the 25 schools included in the study

consecutive days. Stool samples were transferred to nearby laboratories where duplicate Kato-Katz thick smears were prepared on microscope slides from each stool sample. The first set of duplicate slides was examined on the same day under a light microscope, whereas the second set of slides was only examined several days later. The main reason why the second set of slides was examined later was the sheer amount of Kato-Katz thick smears to be examined, coupled with the fact that the study was embedded in a larger SCORE project with particular emphasis on S. mansoni [25]. Slides were examined by experienced laboratory technicians using a standard operating procedure $[27,28]$. All helminth eggs were enumerated and recorded for each species separately. Results from both slides were pooled for each parasite species. Quality control was done on $10 \%$ of the Kato-Katz thick smears.

\section{Questionnaire survey}

Children's knowledge about health-related hygiene, placing particular emphasis on S. mansoni and soil-transmitted helminth infections, their treatment, prevention, symptoms and modes of transmission, were assessed. We also explored children's attitudes and practices regarding hygiene, and probed for cultural beliefs linked to helminth infections. To allow a combination of qualitative and quantitative data, the questionnaire was designed in a semi-structured manner, including a mixture of open- and closed-ended questions. The questionnaire was administered in French, which is the official language of Côte d'Ivoire and is taught in schools. However, there were a few children who did not speak French. For these non-French speaking children, questions were translated by a local interpreter who was part of the survey team. The questionnaire was pre-tested in a school not otherwise involved in the survey to critically determine its suitability regarding duration, content and comprehension. Interviewers were trained and received instructions concerning the questionnaire and its administration. All children were interviewed individually. The administration of the questionnaire usually lasted between 15 and $30 \mathrm{~min}$.

\section{Statistical analysis}

Questionnaire data were double entered and cross-checked in Epilnfo version 3.5.3 (Centers for Disease Control and Prevention; Atlanta, GA, USA). On the spot, parasitological data were entered into smartphones and transferred onto a central server (Open Data Kit) hosted by the SCORE secretariat at the Task Force for Global Health (Decatur, GA, USA) $[25,26]$. The questionnaire and parasitological data were combined into a single master file for subsequent statistical analysis. We used STATA version 10.1 (StataCorp. 2007; College Station, TX, USA) and Quantitative Parasitology version 3.0 [29].

The number of helminth eggs per gram of stool (EPG) was employed as proxy for infection intensity [30]. Infection intensity was grouped into light, moderate and heavy, according to WHO guidelines [30]. 
Factor analysis was used to create two types of scores: (i) a general knowledge score, which expresses children's knowledge regarding helminth infections in general; and (ii) helminth species-specific scores, which estimates the participant's knowledge regarding a specific helminth species. To test whether general knowledge or helminth species-specific scores influenced the risk of infection, a meta-analysis (risk ratio (RR)-based) was performed for each helminth separately as well as for any helminth [31]. Of note, Ascaris lumbricoides and Trichuris trichiura were pooled because of the low prevalence and similar transmission pathways. To assess whether knowledge influenced the intensity of infection, the helminth species-specific median EPG were compared between more and less knowledgeable groups of children, using Kruskal-Wallis tests.

Because the data are clustered (by school), to test whether sex or age group (9-10 years or 11-12 years) influenced how knowledgeable children were, we used generalized estimated equations (GEE). The exchangeable correlation matrix was chosen because the order of sampling of children does not affect the correlation. Since the data are binary (i.e. more knowledgeable and less knowledgeable), a logistic link function was applied. The same method was used to test whether the infection rates were influenced by sex or age group.

Since not all participants replied to the questionnaire and not all provided three stool specimens, two different cohorts will be mentioned throughout the results section. The first cohort consists of all children who responded to the questionnaire but provided less than three stool specimens, and hence, were used when presenting questionnaire results alone. The second cohort consists of those children who had complete data records (i.e. responded to the questionnaire and provided three stool samples), used when testing association between knowledge and infection.

\section{Knowledge scores}

Questionnaire surveys might include questions that are redundant or inadequate to quantify knowledge. Factor analysis is a useful technique to explore which of the questions best measure knowledge [32]. This method allows the identification of the questions that are particularly prone to reveal knowledge, as well as those that should either be discarded because they are uninformative, or pooled to minimize redundancy [33]. Factor analysis loadings represent the level of association between a variable and each factor (for the purpose of our analysis this factor is knowledge) and are used in the final interpretation of the analysis. Higher loadings represent a stronger association between a question and a child's knowledge.

We discarded questions which had loadings lower than 0.4 on the factor representing knowledge. The remaining questions were used to create a summated scale, which only included variables that loaded highly on the factor and excluded those that had little effect. Hence, this scale consisted on the combination of several questions into a single measure of knowledge. We assessed the internal consistency of the summated scale, i.e. whether the questions in the scale had a strong relationship between each other. We used the Cronbach alpha $(\alpha)$, which is a measure of scale reliability. The relationship with the latent variable (knowledge) was considered strong if $\alpha \geq 0.6$ [34]. The results of the factor analysis suggested that several questions should be combined (Table 1). For example, in the case of the symptom question "what are the symptoms of intestinal worms?", the three sub-questions "do they cause diarrhoea?", "do they cause a belly ache?" and "do they cause fatigue?" were combined into a single score. Other questions were combined using the same approach. Overall, six main questions were selected for the construction of this score. In all cases, the participant was considered to have an overall positive answer when he/she replied correctly to over $65 \%$ of the sub-questions. This cut-off was defined as a percentage because each of the six selected questions had a different number of sub-questions making it impossible to define a single number of sub-questions that should be answered correctly in order to be considered knowledgeable regarding that question. The general knowledge score of each participant was calculated by summing the points gained in all six main questions selected by the aforementioned methodology (Table 1). A child was considered to have high general knowledge when he/she replied correctly to at least five of the six questions. This way, participants were separated into two groups based on the results of the questionnaire: good knowledge (five or six correct answers) and less knowledgeable (four or less correct answers).

The questionnaire included a few questions which reflected the interviewee's knowledge regarding the transmission pathway of a specific helminth species. Using these questions, we created three parasite-specific scores: (i) A. lumbricoides and T. trichiura combined; (ii) hookworm; and (iii) S. mansoni. For the A. lumbricoides and T. trichiura combined score, three questions were selected, and hence, a child was considered to have good knowledge if he/she answered correctly to two out of the three questions. With regard to the $S$. mansoni and hookworm scores, a child was considered to have good knowledge if he/she answered correctly to one specific question related to each parasite.

\section{Results}

Operational results

Stool samples were obtained from 2498 children, of whom 1922 (76.9\%) provided three, 472 (18.9\%) two and 
Table 1 Explanation of the general knowledge scoring process

\begin{tabular}{|c|c|c|}
\hline Question & Possible answers & Points \\
\hline \multirow[t]{7}{*}{ Are these correct modes of helminth transmission? } & - Eating without washing hands & \multirow[t]{7}{*}{+1 point if $\geq 5$ questions were answered affirmatively } \\
\hline & - Drinking from the backwater & \\
\hline & - Playing in dirty water & \\
\hline & - Walking on garbage & \\
\hline & - Not wearing shoes & \\
\hline & - Playing in the soil & \\
\hline & - Eating raw vegetables/fruits & \\
\hline \multirow[t]{3}{*}{ Are these incorrect modes of transmission (myths)? } & • Eating too much sugar & \multirow[t]{3}{*}{ - 1 point if $\geq 2$ questions were answered affirmatively } \\
\hline & - Eating too much fruit & \\
\hline & • Eating rotten food & \\
\hline \multirow[t]{7}{*}{ Are these symptoms of intestinal worm infections? } & - Fatigue & \multirow[t]{7}{*}{+1 point if $\geq 5$ questions were answered affirmatively } \\
\hline & - Hard time concentrating & \\
\hline & • Hard time thinking & \\
\hline & • Diarrhoea & \\
\hline & - Not growing well & \\
\hline & - Abdominal pain & \\
\hline & - Lack of appetite & \\
\hline Should you defecate in latrines? & & +1 point if answered affirmatively \\
\hline Should you open air defecate? & & - 1 point if answered affirmatively \\
\hline \multirow[t]{2}{*}{ Should you drink from the backwater? } & & - 1 point if answered affirmatively \\
\hline & & Total general knowledge score \\
\hline
\end{tabular}

The sum of all points was considered for stratification of children into good knowledge ( $\geq 5$ points) or less knowledgeable ( $<5$ points)

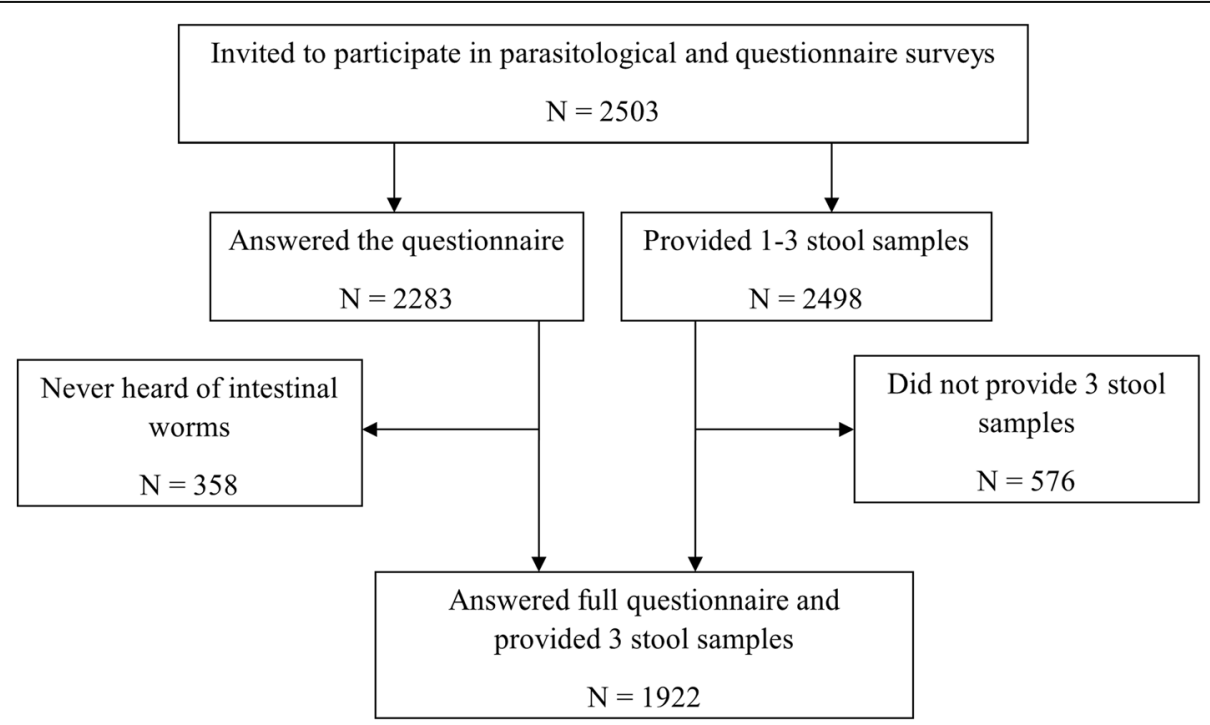

Fig. 2 Participation flow chart. Children who never heard of parasitic worms did not reply to the whole questionnaire and therefore were not included in the analysis related to knowledge 
104 (4.2\%) one stool sample. Questionnaire results were available from 2283 children. However, 358 children (15.7\%) had never heard of parasitic worms, and hence, they were only included in the parasitological analysis (Fig. 2). Of the 1922 children with complete parasitological data, 852 (44.3\%) were females. In terms of age, 1035 children (53.8\%) were aged 9-10 years, while the remaining 887 children (46.2\%) were 11 or 12 years old. The prevalence of hookworm, S. mansoni, T. trichiura and A. lumbricoides was $14.4,8.9,2.6$ and $1.6 \%$, respectively.

\section{Questionnaire survey}

Based on the calculated general knowledge scores, only a third (34.8\%) of the children was considered to have a good knowledge (Table 2).

We found that sex was not associated with knowledge about helminth infections (GEE, $p>0.05$ for any helminth). However, older children were significantly more knowledgeable for each of the four helminth species (GEE, $p<0.001$ for each helminth species). Table 3 shows the percentage of children who answered correctly each of the questions used to create the helminth species-specific scores.

Although not included in the score, the questionnaire revealed that $85 \%$ of children believed that there is efficient treatment available and of those, $86 \%$ believed that it prevents from re-infection.

\section{Influence of knowledge on helminth infections}

Figure 3 depicts the effect of knowledge about modes of helminth transmission on the odds of infection. We found no significant influence of general knowledge on the infection rate by any of the four helminth species (meta-analysis, $p=0.839$ ). We also observed that answering correctly to questions associated with $S$. mansoni did not influence its prevalence (meta-analysis, $p=0.884$ ); hence, children who knew that playing in dirty water is a risk factor for infection showed similar infection rates than those who were less knowledgeable. The same applied to the questions related to A. lumbricoides and T. trichiura (meta-analysis, $p=0.265$ ); children who had a good knowledge about these

Table 2 Percentage of children answering correctly to the general knowledge questions during the cross-sectional survey in 25 schools of western Côte d'Ivoire in May $2014(N=2283)$

\begin{tabular}{lll}
\hline Number of correct answers & \% of children & Knowledge category \\
\hline 0 & 0.3 & Less knowledgeable \\
1 & 2.8 & \\
2 & 9.1 & \\
3 & 13.2 & \\
4 & 39.8 & Good knowledge \\
5 & 30.6 & \\
6 & 4.2 & \\
\hline
\end{tabular}

two helminth species were not found to be less infected. However, in the case of hookworm, specific knowledge that using a latrine for defecation is an appropriate behaviour was associated with a significantly lower odds of hookworm infection (meta-analysis, $p=0.034$ ) (Fig. 3). The forest plot shows that, despite some variability among schools, there is a tendency that a low RR is associated with a higher knowledge level (Fig. 4).

In terms of infection intensity, we found that knowledge about a specific helminth species was not associated with an overall lower level of infection; hence, children who answered correctly to questions related to specific helminth species were not infected with a lower intensity than those who did not answer correctly (Kruskal-Wallis, $p>0.05$ for any helminth). The same observation was made for general knowledge; higher general knowledge scores were not associated with lighter infection intensities with any helminth (Kruskal-Wallis, $p>0.05$ ).

\section{Discussion}

Children with good hygiene knowledge (e.g. use of latrine for defecation) had lower hookworm prevalence than their counterparts who lacked such knowledge. It is conceivable that children who perceive open defecation as a negative behaviour are more likely to use latrines. Such children might also more often wear shoes, and hence, are less prone to stepping on contaminated soil while visiting areas that are convenient to defecate near villages. Our results are encouraging and in line with experiences from a health education intervention gained in the People's Republic of China [17]. Indeed, the implementation of an animated cartoon had a positive effect on children's behaviour; it reduced soil-transmitted helminth infections by $50 \%$ demonstrating that, at least in some contexts, better knowledge can effectively improve children's behaviour. However, in the current study, although we found a significant relationship between hygiene knowledge and hookworm infection, children who scored higher in general knowledge had similar infection prevalence of $S$. mansoni and A. lumbricoides-T. trichiura combined as their less knowledgeable counterparts. Our findings suggest that knowledge is not necessarily effective in inducing changes in behaviour and health-related practices that prevent helminth infection. Hence, even when children knew how to avoid helminth infections, they did not necessarily change their hygiene- and health-related practices. Other groups have also described situations in which acquiring knowledge did not readily translate into behavioural changes [35-38].

How can these observations be explained? First, children may underestimate the risk associated with helminth infections because they are not fully aware of their potential severity or of their long term consequences. In fact, most of the children interviewed (85\%) 
Table 3 Percentage of children answering correctly to each of the questions used for the parasite-specific scores during a crosssectional survey in 25 schools of western Côte d'Ivoire in May $2014(N=2283)$

\begin{tabular}{lll}
\hline Helminth infection & Question & \% of children answering correctly \\
\hline S. mansoni & Can you become infected if you play in dirty water? & 61.8 \\
Hookworm & Should you defecate in latrines? & 82.5 \\
A. lumbricoides and T. trichiura combined & Can you become infected if you do not wash your & 65.2 \\
& hands before eating? & 45.5 \\
& Can you become infected if you drink water from the river? & 55.4 \\
\hline
\end{tabular}

believed that efficacious treatment is available and that, once treated, they cannot become re-infected (86\%). Such a misconception, which may result from confusion with the long-term protection by vaccination, might lower the perceived risk associated with helminth infections, and hence, reducing the incentive to avoid risky behaviours. Overcoming such misconceptions is, therefore, critical to initiate a change in behaviour. The way knowledge is being transmitted to children does not seem to properly convey the potential danger of these infections, and hence, does not instigate great urge to correctly apply it. The benefits and shortcomings of preventive chemotherapy should be explained more thoroughly in school health programmes. It may be of value to encourage teachers to stimulate children, on a frequent basis, to apply their knowledge as well as remind them of the consequences of not doing so.

Second, the fact that children do not necessarily change their hygiene- and health-related behaviour in response to a deeper understanding of the epidemiology of helminthiases might be explained by a lack of access to clean water and improved sanitation, which obviously

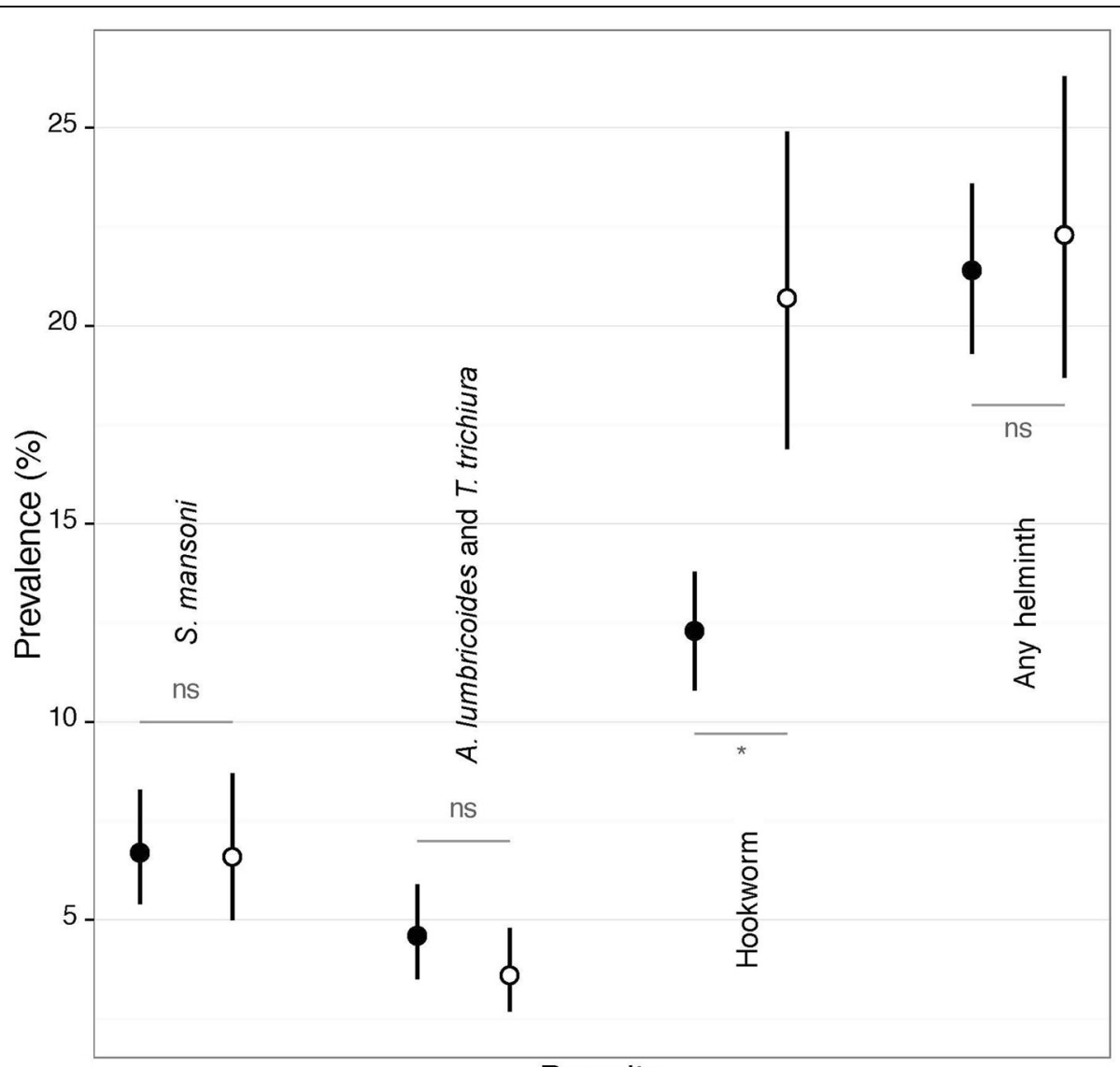

Parasite

Fig. 3 Prevalence of helminth infection in children with more (black dot) and less (white dot) knowledge of the modes of transmission of each helminth species. $\mathrm{ns}=$ non-significant, ${ }^{*}=P<0.05$ 


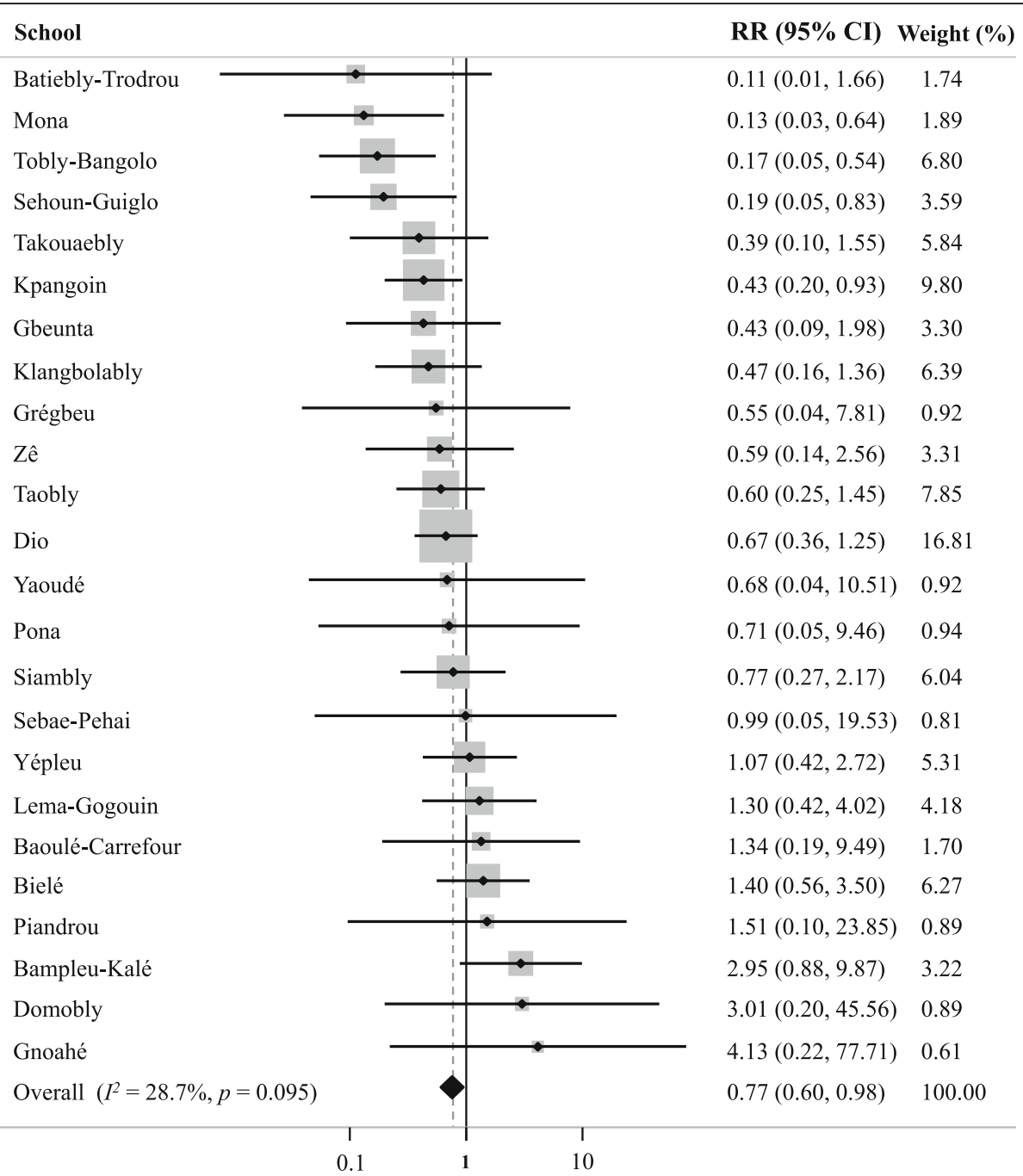

Fig. 4 Forest plot representing the overall and school-level effects of knowledge about hookworm on the infection RR

represents a barrier between knowledge and practice. For instance, even if children are aware of the risks of getting into contact with contaminated water sites, they will continue to do so until there are alternatives, as shown by Schall [36]. Children are unlikely to give up recreational bathing and playing in a river or a lake on a hot day just because someone told them they may become sick. A study by Kosinski and colleagues in Ghana included the construction of a safe water recreation area [39]. One year after the installation of this water recreation area, the prevalence of $S$. haematobium in children had decreased significantly, illustrating the importance of providing a safe alternative for recreational water activities, in addition to improved knowledge. Increasing the population's knowledge and wish to improve hygiene and health-related behaviours without providing the conditions to put the new knowledge into practice may disempower and frustrate people [38]. Hence, efforts are warranted to ensure that the recommended behaviours can be practiced. In schools, it is important to find strategies that make teachers feel responsible and motivate them to ensure there is always easily available water and soap in their schools and that latrines are clean and being used correctly. Still, it is important to note that the simple provision of sanitation facilities is not always sufficient. Studies have illustrated situations where available facilities were not widely used, and hence, were not associated with lower helminth infection rates [11]. Whenever facilities are available, helminth infections will depend on consistent use and good maintenance of the facilities [14]. Indeed, in Zambia, Thys and colleagues found that bad smell, presence of flies, inadequate maintenance and challenges of waste disposal explained a continuing preference for open defecation [40].

Third, unlike prior research in the People's Republic of China [17], we did not find a consistent link between 
knowledge and helminth infection rates. In our study in Côte d'Ivoire, deeper knowledge did not automatically translate into a decrease of helminth infections. Social and cultural differences may play a role in how well behaviour change is achieved and should, thus, be taken into account in the design of health education interventions. Social pressure may be a strategy to motivate children to start following the health and hygiene-related recommendations more often. Gyorkos and colleagues suggested that social marketing at the community level as well as further involvement of teachers and parents could potentially maximize changes in behaviour leading to a reduction of infection rates [20].

\section{Strengths and limitations of the study}

Unlike previous studies, which aimed at determining the effect of health education interventions on knowledge and helminth infection, the present study focused on the influence of background knowledge on helminth infection. This unique perspective allowed us to identify the type of knowledge, which is most associated with soil-transmitted helminths and $S$. mansoni and, thus, reinforce setting-specific messages for health education interventions. Our study also contributed to a deeper understanding of strategies that are insufficiently effective, and hence, must be reinforced by other strategies. An additional strength of our study resides in the creation of a general knowledge score. In order to identify the most suitable questions for assessing knowledge, we pursued a factor analysis. This method identifies those questions that should not be included and turns the score into a more reliable one.

A limitation of our study is related to the low prevalence of some helminth species, most importantly A. lumbricoides and T. trichiura. We employed repeated stool sampling and prepared two Kato-Katz thick smears from each stool specimen to increase diagnostic sensitivity, and yet, the prevalence of both $A$. lumbricoides and T. trichiura was below $3 \%$. Of note, from the duplicate Kato-Katz thick smears produced, only one was microscopically examined the same day, whilst the second slide was examined only after several days. Hence, the true prevalence of hookworm might have been underestimated, not only because all infections were of light intensity and therefore remained more likely undetected compared to moderate and heavy infections [41], but also because hookworm eggs tend to disintegrate rapidly after preparation of thick smears. Nonetheless, we found a significant association between knowledge and infection rate with hookworm.

\section{Conclusions}

Taken together, children's knowledge of S. mansoni, T. trichiura and A. lumbricoides varied, yet the infection rates of the most knowledgeable children were similar to less knowledgeable children. However, we showed that children who were aware of the risk of open defecation were less likely to be infected with hookworm. This result is encouraging, as it suggests that knowledge has the potential to translate into behavioural changes that reduce the risk of infection. Being the main source of most health education messages that children receive, we find that schools should (1) provide an opportunity to apply this health and hygiene-related knowledge and (2) increase children's awareness and willingness to improve their hygiene behaviours. If schools fail to fulfil one or both of these two key functions, which is often the case, they will inevitably diminish the school's potential to improve children's health. Education initiatives to complement the role of schools may gain from prior evaluation of the level of knowledge of children. In situations like that of our study, where children already had a fair background knowledge, it may be advantageous to concentrate most of those efforts on implementing strategies to convert knowledge into behavioural changes. As a next logical step we suggest concrete activities to improve preventive behaviour for the other parasites in order to decrease their infection rates.

\section{Abbreviations}

EPG: Eggs per gram of stool; GEE: Generalized estimating equations; RR: Risk ratio; SCORE: Schistosomiasis Consortium for Operational Research and Evaluation; WHO: World Health Organization

\section{Acknowledgements}

We thank the authorities and inhabitants of the study villages and particularly the schoolchildren for their enthusiastic participation. We are grateful to all the local field assistants, laboratory technicians and drivers from the Centre Suisse de Recherches Scientifiques en Côte d'Ivoire for their dedicated and hard work during the parasitological and questionnaire surveys.

\section{Funding}

This study received financial support from the UBS Optimus Foundation and the University of Georgia Research Foundation Inc., which is funded by the Bill \& Melinda Gates Foundation for these Schistosomiasis Consortium for Operational Reserach and Evaluation (SCORE) projects (prime award no 50816; sub-award no. RR374-053/4893196). The funders had no role in study design, data collection and analysis, decision to publish, or preparation of the manuscript.

\section{Availability of data and materials}

The datasets used and/or analysed during the current study are available from the corresponding author upon reasonable request.

\section{Author's contributions}

JU, EKN and GR designed the study; MO, CE, VAK, RKA, EH, JTC, NRD and KD implemented the study; MO, RKA and EH managed the data; MSP analysed the data and wrote the first draft of the paper; JU and GR contributed to data analysis and helped interpreting the results; MSP, BB, JU and GR revised the manuscript and provided important intellectual content. All authors read and approved the final version of the manuscript before submission.

\section{Ethics approval and consent to participate}

Ethical clearance for the study was obtained by the Ethics Committee of Basel, Switzerland (EKBB; reference no. 300/13, date of approval: 11

November 2013) and from the ethics committee of the Ministry of Health and Public Hygiene in Côte d'Ivoire (reference no. 76-MSLS-CNER-dkn, date of approval: 28 November 2013). Written informed consent was obtained from the parents or guardians of participating children, while children assented orally. 


\section{Consent for publication}

Not applicable.

\section{Competing interests}

The authors declare they have no competing interests.

\section{Publisher's Note}

Springer Nature remains neutral with regard to jurisdictional claims in published maps and institutional affiliations.

\section{Author details}

${ }^{1}$ Swiss Tropical and Public Health Institute, Basel, Switzerland. ${ }^{2}$ University of Basel, Basel, Switzerland. ${ }^{3}$ Institute of Hygiene and Tropical Medicine, New University of Lisbon, Lisbon, Portugal. ${ }^{4}$ Unité de Formation et de Recherche Biosciences, Université Félix Houphouët-Boigny, Abidjan, Côte d'Ivoire. ${ }^{5}$ Unité de Formation et de Recherche Sciences de l'Homme et de la Société, Université Félix Houphouët-Boigny, Abidjan, Côte d'Ivoire. ${ }^{6}$ Centre Suisse de Recherches Scientifiques en Côte d'Ivoire, Abidjan, Côte d'Ivoire.

\section{Received: 28 November 2017 Accepted: 28 June 2018}

\section{Published online: 02 August 2018}

\section{References}

1. WHO. Summary of global update on preventive chemotherapy implementation in 2015. Wkly Epidemiol Rec. 2016;91(39):456-9.

2. Pullan $\mathrm{RL}$, Smith JL, Jasrasaria $\mathrm{R}$, Brooker SJ. Global numbers of infection and disease burden of soil transmitted helminth infections in 2010. Parasit Vectors. 2014;7:37.

3. King CH, Dickman K, Tisch DJ. Reassessment of the cost of chronic helmintic infection: a meta-analysis of disability-related outcomes in endemic schistosomiasis. Lancet. 2005;365(9470):1561-9.

4. Bethony J, Brooker S, Albonico M, Geiger SM, Loukas A, Diemert D, Hotez PJ. Soil-transmitted helminth infections: ascariasis, trichuriasis, and hookworm. Lancet. 2006;367(9521):1521-32.

5. Hotez PJ, Molyneux DH, Fenwick A, Kumaresan J, Ehrlich Sachs S, Sachs JD, Savioli L. Control of neglected tropical diseases. N Engl J Med. 2007;357:1018-27.

6. Utzinger J, Becker SL, Knopp S, Blum J, Neumayr AL, Keiser J, Hatz CF. Neglected tropical diseases: diagnosis, clinical management, treatment and control. Swiss Med Wkly. 2012;142:w13727.

7. Hotez PJ, Alvarado M, Basáñez MG, Bolliger I, Bourne R, Boussinesq M, Brooker SJ, Brown AS, Buckle G, Budke CM, et al. The Global Burden of Disease study 2010: interpretation and implications for the neglected tropical diseases. PLoS Negl Trop Dis. 2014;8(10):e2865.

8. GBD 2015 DALYs and HALE Collaborators. Global, regional, and national disability-adjusted life-years (DALYS) for 315 diseases and injuries and healthy life expectancy (HALE), 1990-2015: a systematic analysis for the Global Burden of Disease study 2015. Lancet. 2016;388(10053):1603-58.

9. WHO. Deworming for health and development: report of the third global meeting of the Partners for Parasite Control. Geneva: World Health Organization; 2005.

10. Jia TW, Melville S, Utzinger J, King CH, Zhou XN. Soil-transmitted helminth reinfection after drug treatment: a systematic review and meta-analysis. PLoS Negl Trop Dis. 2012;6(5):e1621.

11. Patil SR, Arnold BF, Salvatore AL, Briceno B, Ganguly S, Colford JM Jr, Gertler PJ. The effect of India's total sanitation campaign on defecation behaviors and child health in rural Madhya Pradesh: a cluster randomized controlled trial. PLoS Med. 2014;11(8):e1001709.

12. Strunz EC, Addiss DG, Stocks ME, Ogden S, Utzinger J, Freeman MC. Water sanitation, hygiene, and soil-transmitted helminth infection: a systematic review and meta-analysis. PLoS Med. 2014;11(3):e1001620.

13. Albonico M, Smith PG, Ercole E, Hall A, Chwaya HM, Alawi KS, Savioli L. Rate of reinfection with intestinal nematodes after treatment of children with mebendazole or albendazole in a highly endemic area. Trans R Soc Trop Med Hyg. 1995;89(5):538-41.

14. Asaolu SO, Ofoezie IE. The role of health education and sanitation in the control of helminth infections. Acta Trop. 2003:86(2-3):283-94.

15. Mascarini-Serra L. Prevention of soil-transmitted helminth infection. J Glob Infect Dis. 2011;3:175-82

16. Gazzinelli A, Correa-Oliveira R, Yang GJ, Boatin BA, Kloos H. A research agenda for helminth diseases of humans: social ecology, environmental determinants, and health systems. PLoS Negl Trop Dis. 2012;6(4):e1603.
17. Bieri FA, Gray DJ, Williams GM, Raso G, Li YS, Yuan L, He Y, Li RS, Guo FY, Li SM, et al. Health-education package to prevent worm infections in Chinese schoolchildren. N Engl J Med. 2013;368(17):1603-12.

18. Albright JW, Basaric-Keys J. Instruction in behavior modification can significantly alter soil-transmitted helminth (STH) re-infection following therapeutic de-worming. Southeast Asian J Trop Med Public Health. 2006;37(1):48-57.

19. Anantaphruti MT, Waikagul J, Maipanich W, Nuamtanong S, Watthanakulpanich D, Pubampen S, Kusolsuk T. School-based health education for the control of soil-transmitted helminthiases in Kanchanaburi province, Thailand. Ann Trop Med Parasitol. 2008;102(6):521-8.

20. Gyorkos TW, Maheu-Giroux M, Blouin B, Casapia M. Impact of health education on soil-transmitted helminth infections in schoolchildren of the Peruvian Amazon: a cluster-randomized controlled trial. PLoS Negl Trop Dis. 2013;7(9):e2397.

21. Al-Delaimy AK, Al-Mekhlafi HM, Lim YA, Nasr NA, Sady H, Atroosh WM, Mahmud R. Developing and evaluating health education learning package (HELP) to control soil-transmitted helminth infections among Orang Asli children in Malaysia. Parasit Vectors. 2014;7:416.

22. Utzinger J, N'Goran EK, Ossey YA, Booth M, Traoré M, Lohourignon KL, Allangba A, Ahiba LA, Tanner M, Lengeler C. Rapid screening for Schistosoma mansoni in western Côte d'Ivoire using a simple school questionnaire. Bull World Health Organ. 2000;78(3):389-98.

23. Keiser J, N'Goran EK, Traoré M, Lohourignon KL, Singer BH, Lengeler C, Tanner M, Utzinger J. Polyparasitism with Schistosoma mansoni, geohelminths, and intestinal protozoa in rural Côte d'Ivoire. J Parasitol. 2002;88(3):461-6.

24. Raso G, Matthys B, N'Goran EK, Tanner M, Vounatsou P, Utzinger J. Spatial risk prediction and mapping of Schistosoma mansoni infections among schoolchildren living in western Côte d'Ivoire. Parasitology. 2005;131(Pt 1):97-108.

25. Assaré RK, Knopp S, N'Guessan NA, Yapi A, Tian-Bi YNT, Yao PK, Coulibaly JT, Ouattara M, Meïté A, Fenwick A, et al. Sustaining control of schistosomiasis mansoni in moderate endemicity areas in western Côte d'Ivoire: a SCORE study protocol. BMC Public Health. 2014;14:1290.

26. Ezeamama $A E, H e C L$, Shen $Y$, Yin XP, Binder SC, Campbell CH Jr, Rathbun $S$, Whalen CC, N'Goran EK, Utzinger J, et al. Gaining and sustaining schistosomiasis control: study protocol and baseline data prior to different treatment strategies in five African countries. BMC Infect Dis. 2016;16:229.

27. Katz N, Chaves A, Pellegrino J. A simple device for quantitative stool thicksmear technique in schistosomiasis mansoni. Rev Inst Med Trop São Paulo. 1972;14(6):397-400.

28. Becker SL, Yap P, Horie NS, Alirol E, Barbe B, Bhatta NK, Bhattarai NR, Bottieau E, Chatigre JK, Coulibaly JT, et al. Experiences and lessons from a multicountry NIDIAG study on persistent digestive disorders in the tropics. PLoS Negl Trop Dis. 2016;10(11):e0004818.

29. Rozsa L, Reiczigel J, Majoros G. Quantifying parasites in samples of hosts. J Parasitol. 2000;86(2):228-32.

30. WHO. Helminth control in school age children: a guide for managers of control programmes. Geneva: World Health Organization; 2011.

31. Reiczigel J. Confidence intervals for the binomial parameter: some new considerations. Stat Med. 2003;22(4):611-21.

32. Chateau D, Metge C, Prior H, Soodeen RA. Learning from the census: the socio-economic factor index (SEFI) and health outcomes in Manitoba. Can J Public Health. 2012;103(8 Suppl 2):S23-7.

33. Hair JF, Black WC, Babin BJ, Anderson RE, Tatham RL. Multivariate data analysis. 5th ed. Upper Saddle River: Pearson Prentice Hall; 1998.

34. Sim K, Wright C. Research in health care: concepts, designs and methods. Cheltenham: Stanley Thornes Ltd; 2000.

35. Quick RE, Gerber ML, Palacios AM, Beingolea L, Vargas R, Mujica O, Moreno D, Seminario L, Smithwick EB, Tauxe RV. Using a knowledge, attitudes and practices survey to supplement findings of an outbreak investigation: cholera prevention measures during the 1991 epidemic in Peru. Int J Epidemiol. 1996;25(4):872-8.

36. Schall VT. An interactive perspective of health education for the tropical disease control: the schistosomiasis case. Mem Inst Oswaldo Cruz. 1998;93(Suppl 1):51-8

37. Acka CA, Raso G, N'Goran EK, Tschannen AB, Bogoch II, Seraphin E, Tanner M, Obrist B, Utzinger J. Parasitic worms: knowledge, attitudes, and practices in western Côte d'Ivoire with implications for integrated control. PLoS Negl Trop Dis. 2010;4(12):e910.

38. Fisher S, Kabir B, Lahiff E, Maclachlan M. Knowledge, attitudes, practices and implications of safe water management and good hygiene in rural 
Bangladesh: assessing the impact and scope of the BRAC WASH programme. J Water Health. 2011;9(1):80-93.

39. Kosinski KC, Adjei MN, Bosompem KM, Crocker JJ, Durant JL, Osabutey D, Plummer JD, Stadecker MJ, Wagner AD, Woodin M, et al. Effective control of Schistosoma haematobium infection in a Ghanaian community following installation of a water recreation area. PLoS Negl Trop Dis. 2012;6(7):e1709.

40. Thys S, Mwape KE, Lefevre P, Dorny P, Marcotty T, Phiri AM, Phiri IK, Gabriel $S$. Why latrines are not used: communities' perceptions and practices regarding latrines in a Taenia solium endemic rural area in eastern Zambia. PLoS Negl Trop Dis. 2015;9(3):e0003570.

41. Ngui R, Ching LS, Kai TT, Roslan MA, Lim YA. Molecular identification of human hookworm infections in economically disadvantaged communities in peninsular Malaysia. Am J Trop Med Hyg. 2012;86(5):837-42.

- fast, convenient online submission

- thorough peer review by experienced researchers in your field

- rapid publication on acceptance

- support for research data, including large and complex data types

- gold Open Access which fosters wider collaboration and increased citations

- maximum visibility for your research: over $100 \mathrm{M}$ website views per year 